\title{
Discovery of concentric broken rings at sub-arcsec separations in the HD 141569A gas-rich, debris disk with VLT/SPHERE`
}

\author{
C. Perrot ${ }^{1}$, A. Boccaletti ${ }^{1}$, E. Pantin ${ }^{2}$, J.-C. Augereau ${ }^{3,4}$, A.-M. Lagrange ${ }^{3,4}$, R. Galicher ${ }^{1}$, A.-L. Maire ${ }^{5}$, J. Mazoyer ${ }^{6}$,
} J. Milli ${ }^{7}$, G. Rousset ${ }^{1}$, R. Gratton ${ }^{8}$, M. Bonnefoy ${ }^{3,4}$, W. Brandner ${ }^{5}$, E. Buenzli ${ }^{9}$, M. Langlois ${ }^{10}$, J. Lannier ${ }^{3,4}$,

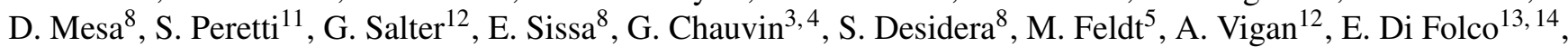
A. Dutrey ${ }^{13,14}$, J. Péricaud ${ }^{13,14}$, P. Baudoz ${ }^{1}$, M. Benisty ${ }^{3,4}$, J. De Boer ${ }^{15}$, A. Garufi ${ }^{9}$, J. H. Girard ${ }^{7}$, F. Menard ${ }^{3,4}$, J. Olofsson ${ }^{5,16,17}$, S. P. Quanz ${ }^{9}$, D. Mouillet ${ }^{3,4}$, V. Christiaens ${ }^{18,19}$, S. Casassus ${ }^{18}$, J.-L. Beuzit ${ }^{3,4}$, P. Blanchard ${ }^{12}$, M. Carle ${ }^{12}$, T. Fusco ${ }^{20,12}$, E. Giro ${ }^{8}$, N. Hubin ${ }^{21}$, D. Maurel ${ }^{3,4}$, O. Moeller-Nilsson ${ }^{5}$, A. Sevin ${ }^{1}$, and L. Weber ${ }^{11}$

${ }^{1}$ LESIA, Observatoire de Paris, PSL Research Univ., CNRS, Univ. Paris Diderot, Sorbonne Paris Cité, UPMC Paris 6 , Sorbonne Univ., 5 place Jules Janssen, 92195 Meudon Cedex, France

e-mail: clement.perrot@obspm.fr

2 Laboratoire AIM, CEA/DSM - CNRS - Univ. Paris Diderot, IRFU/SAp, 91191 Gif-sur-Yvette, France

3 Univ. Grenoble Alpes, IPAG, 38000 Grenoble, France

4 CNRS, IPAG, 38000 Grenoble, France

5 Max-Planck-Institut für Astronomie, Königstuhl 17, 69117 Heidelberg, Germany

${ }^{6}$ Space Telescope Science Institute, 3700 San Martin Dr, Baltimore MD 21218, USA

7 European Southern Observatory, Alonso de Cordova 3107, Casilla 19001 Vitacura, Santiago 19, Chile

8 INAF - Osservatorio Astronomico di Padova, Vicolo dell'Osservatorio 5, 35122 Padova, Italy

9 Institute for Astronomy, ETH Zurich, Wolfgang-Pauli-Strasse 27, 8093 Zurich, Switzerland

${ }^{10}$ CNRS/CRAL/Observatoire de Lyon/Univ. de Lyon 1/École Normale Supérieure de Lyon, Lyon, France

11 Geneva Observatory, Univ. of Geneva, Ch. des Maillettes 51, 1290 Versoix, Switzerland

12 Aix-Marseille Univ., CNRS, LAM - Laboratoire d'Astrophysique de Marseille, UMR 7326, 13388 Marseille, France

13 Univ. Bordeaux, Laboratoire d'Astrophysique de Bordeaux, UMR 5804, 33270 Floirac, France

14 CNRS, LAB, UMR 5804, 33270 Floirac, France

15 Leiden Observatory, Leiden Univ., PO Box 9513, 2300 RA Leiden, The Netherlands

16 Instituto de Física y Astronomía, Facultad de Ciencias, Univ. de Valparaíso, Av. Gran Bretaña 1111, Playa Ancha, Valparaíso, Chile

17 ICM nucleus on protoplanetary disks, Univ. de Valparaíso, Av. Gran Bretaña 1111, Valparaíso, Chile

18 Departamento de Astronomía, Universidad de Chile, Casilla 36-D, Santiago, Chile

19 Département d'Astrophysique, Géophysique et Océanographie, Univ. de Liège, Allée du Six Août 17, 4000 Liège, Belgique

20 ONERA, The French Aerospace Lab BP72, 29 avenue de la Division Leclerc, 92322 Châtillon Cedex, France

21 European Southern Observatory, Karl Schwarzschild St., 2, 85748 Garching, Germany

Received 26 February 2016/ Accepted 21 April 2016

\section{ABSTRACT}

Context. Transition disks correspond to a short stage between the young protoplanetary phase and older debris phase. Along this evolutionary sequence, the gas component disappears leaving room for a dust-dominated environment where already-formed planets signpost their gravitational perturbations.

Aims. We endeavor to study the very inner region of the well-known and complex debris, but still gas-rich disk, around HD 141569A using the exquisite high-contrast capability of SPHERE at the VLT. Recent near-infrared (IR) images suggest a relatively depleted cavity within $\sim 200 \mathrm{au}$, while former mid-IR data indicate the presence of dust at separations shorter than $\sim 100$ au.

Methods. We obtained multi-wavelength images in the near-IR in $J, \mathrm{H} 2, \mathrm{H} 3$ and $K$ s-bands with the IRDIS camera and a $0.95-1.35 \mu \mathrm{m}$ spectral data cube with the IFS. Data were acquired in pupil-tracking mode, thus allowing for angular differential imaging.

Results. We discovered several new structures inside $1^{\prime \prime}$, of which the most prominent is a bright ring with sharp edges (semi-major axis: $0.4^{\prime \prime}$ ) featuring a strong north-south brightness asymmetry. Other faint structures are also detected from $0.4^{\prime \prime}$ to $1^{\prime \prime}$ in the form of concentric ringlets and at least one spiral arm. Finally, the VISIR data at $8.6 \mu \mathrm{m}$ suggests the presence of an additional dust population closer in. Besides, we do not detect companions more massive than 1-3 mass of Jupiter.

Conclusions. The performance of SPHERE allows us to resolve the extended dust component, which was previously detected at thermal and visible wavelengths, into very complex patterns with strong asymmetries; the nature of these asymmetries remains to be understood. Scenarios involving shepherding by planets or dust-gas interactions will have to be tested against these observations.

Key words. stars: individual: HD 141569A - protoplanetary disks - planet-disk interactions - stars: early-type techniques: high angular resolution - techniques: image processing 


\section{Introduction}

Observing the short phase of transition between gas-rich protoplanetary disks and dust-dominated debris disks is crucial to constrain the time when planets start to form as well as the environmental conditions. HD 141569A is a young (5 Myr; Merìn et al. 2004) Herbig Ae/Be star classified as A0Ve star $(V=7.12, H=$ $6.861, K=6.821$ ), which is located at $116_{-8}^{+9}$ pc (van Leeuwen $2007)^{1}$. An optically thin disk was resolved in scattered light with Hubble Space Telescope (HST), in the near-IR, as a tworing system located at about $\sim 250$ au and $\sim 410$ au from the star (Augereau et al. 1999a; Weinberger et al. 1999). Using HST in the visible, both Mouillet et al. (2001) and Clampin et al. (2003) observed a more complex environment made of multiple rings and outer spirals, whose presence could be the result of an interaction with two visual stellar companions to HD 141569A (Augereau \& Papaloizou 2004; Ardila et al. 2005), with outer planets (Wyatt 2005), or both (Reche et al. 2009). From the ground, high contrast images in the near-IR were obtained with Near-Infrared Coronagraphic Imager (NICI; Biller et al. 2015; Mazoyer et al. 2016), which started to probe the very inner part of the disk inside the formerly known, innermost ring at $\sim 250 \mathrm{au}$.

While many of the structures observed in the dust distribution are representative of debris disks, HD 141569A also contains a large amount of gas (Brittain et al. 2003; Dent et al. 2005). Thi et al. (2014) show that the gas component detected with the Herschel's PACS instrument in OI and CII cooling lines remains a major component in an hybrid disk such as HD 141569A. At longer wavelengths, the CO gas component has been resolved with the IRAM's Plateau de Bure interferometer (Péricaud et al. 2015) and implies a large amount of cold gas, extending out to a radius of $250 \mathrm{au}$.

The inner region of the disk, inside $\sim 100 \mathrm{au}$, is poorly known and is obviously of great importance when it comes to studying planetary formation and disk evolution. Several observational facts indicate the presence of an inner dust population. First of all, the spectral energy distribution shows an IR excess at $10 \mu \mathrm{m}$ (Thi et al. 2014) and a significant fraction of the total IR disk luminosity arises from regions closer than 100 au (Augereau et al. 1999a). Secondly, a resolved polycyclic aromatic hydrocarbons emission feature localized within $\sim 50$ au and about six times brighter than the expected stellar flux, has been resolved with the VLT Imager and Spectrometer for mid-InfraRed (VISIR) at $8.6 \mu \mathrm{m}$ (Thi et al. 2014). In addition, a CO gas emission line is also resolved within $\sim 50$ au by Goto et al. (2006) with an inner clearing cavity inside $10 \mathrm{au}$. The gas kinematics indicates that the central part of the disk rotates clockwise and the southeast side is in the front, in agreement with what is inferred from the outer part (Dutrey et al. 2004). Attempts in scattered light with differential polarimetry was unsuccessful (Garufi et al. 2014, for instance). But very recently, Konishi et al. (2016) finally detected an extended disk component in the range 46-116 au, corroborated by the north-south emission reported by Currie et al. (2016) in the $L^{\prime}$-band $(3.778 \mu \mathrm{m})$ and located at 30-40 au.

This paper presents the first Spectro-Polarimetric Highcontrast Exoplanet REsearch (SPHERE) observations of the HD 141569A system angularly resolving the scattered light emission inside 200 au in the form of several ringlets and spirals. Section 2 describes the observations and data reduction. We successively present the morphology of the newly resolved

\footnotetext{
* Based on data collected at the European Southern Observatory, Chile, ESO programs 095.C-0381 and 095.C-0298.

1 All distances and radii in this paper are given assuming this revised star distance $(100$ mas $=11.6 \mathrm{au})$.
}

structures (Sect. 3), photometry of the brightest ringlet and a comparison with VISIR data (Sect. 4), and detection limits of point-sources (Sect. 5).

\section{Observations and data reduction}

The extreme adaptive optics coronagraphic instrument SPHERE (Beuzit et al. 2008; Fusco et al. 2014) installed at the VLT in 2014 , is dedicated to the search and characterization of young planetary systems. HD 141569A was observed on May 2015, as part of guaranteed time observation (GTO), using the Dual Band Imaging mode (DBI; Vigan et al. 2010) of the Infra-Red Dualbeam Imager and Spectrograph (IRDIS; Dohlen et al. 2008), with filters $\mathrm{H} 2$ and $\mathrm{H} 3$. Simultaneously, a spectral data cube was obtained with the near-IR Integral Field Spectrograph (IFS; Claudi et al. 2008) in YJ mode (0.95-1.35 $\mu \mathrm{m}$, in 39 channels). A second observation in open time (095.C-0381) was performed on July 2015 with IRDIS in classical imaging (CI; Langlois et al. 2014) in broadband filters $J$ and $K$ s (Table A.1). All observations were obtained with the Apodized Lyot Coronagraph (mask diameter: 185 mas, Boccaletti et al. 2008). Conditions were good for $\mathrm{H} 2 \mathrm{H} 3, \mathrm{YJ}$, and $K$ s-bands and rather poor for $J$-band $\left(\tau_{0}^{2}: 3.5\right.$, 1.1 and 0.9 ms, seeing: $0.76^{\prime \prime}, 1.36^{\prime \prime}$ and $1.28^{\prime \prime}$, respectively for $\mathrm{H} 2 \mathrm{H} 3-\mathrm{YJ}, \mathrm{Ks}$ and $J$ ). IRDIS has a pixel size of $12.25 \pm 0.02$ mas and a field-of-view (FoV) of $11^{\prime \prime} \times 12.5^{\prime \prime}$. IFS pixel size is $7.46 \pm 0.02$ mas for a $1.73^{\prime \prime} \times 1.73^{\prime \prime}$ FoV. The field orientation of IRDIS and IFS are derived from astrometric calibrations as described in Maire et al. (2016). True North corrections are given in Table A.1.

All the data were reduced with the SPHERE pipeline (Pavlov et al. 2008) implemented at the SPHERE Data Center together with additional tools. This includes dark and sky subtraction, bad-pixels removal, flat-field correction, anamorphism correction (Maire et al. 2016), and wavelength calibration ${ }^{3}$. The location of the star is identified with the four symmetrical satellite spots generated from a waffle pattern on the deformable mirror (Marois et al. 2006b). Then, to remove the stellar halo and to achieve high contrast, the data were processed with two highlevel processing pipelines: SpeCal, which was developed for the SPHERE survey (R. Galicher, priv. comm.), and the processing pipeline from our team (Boccaletti et al. 2015), both leading to very similar results. We used a variety of Angular Differential Imaging algorithms: cADI (Marois et al. 2006a), LOCI (Lafrenière et al. 2007), TLOCI (Marois et al. 2014), and KLIP (Soummer et al. 2012). IFS spectral frames were collapsed to increase the signal-to-noise ratio $(\mathrm{S} / \mathrm{N})$.

\section{Morphology of the inner disk}

\subsection{Structures identification}

The disk is oriented at a position angle PA $=356.1 \pm 0.4^{\circ}$ and the inclination is $56.9 \pm 1.0^{\circ}$ (Table A.2); these values are in agreement with Mazoyer et al. (2016). The best images are provided by the IRDIS instrument in $\mathrm{H} 2 \mathrm{H} 3$ (sum of $\mathrm{H} 2$ and H3 images), which best compromises contrast and sensitivity (Fig. 1). All features, recently identified in Biller et al. (2015) and Mazoyer et al. (2016) are recovered at all bands (Fig. B.1, a): 1) the outer ring at a semi-major axis of $3.55^{\prime \prime}(\sim 410 \mathrm{au})$; 2) a very complex belt at $2.20^{\prime \prime}(\sim 255 \mathrm{au})$ split in two parts,

\footnotetext{
2 Coherence time of the atmospheric turbulence at $0.5 \mu \mathrm{m}$.

For the IFS spectral channels as well as the transmissions of both IFS and IRDIS spectral channels.
} 

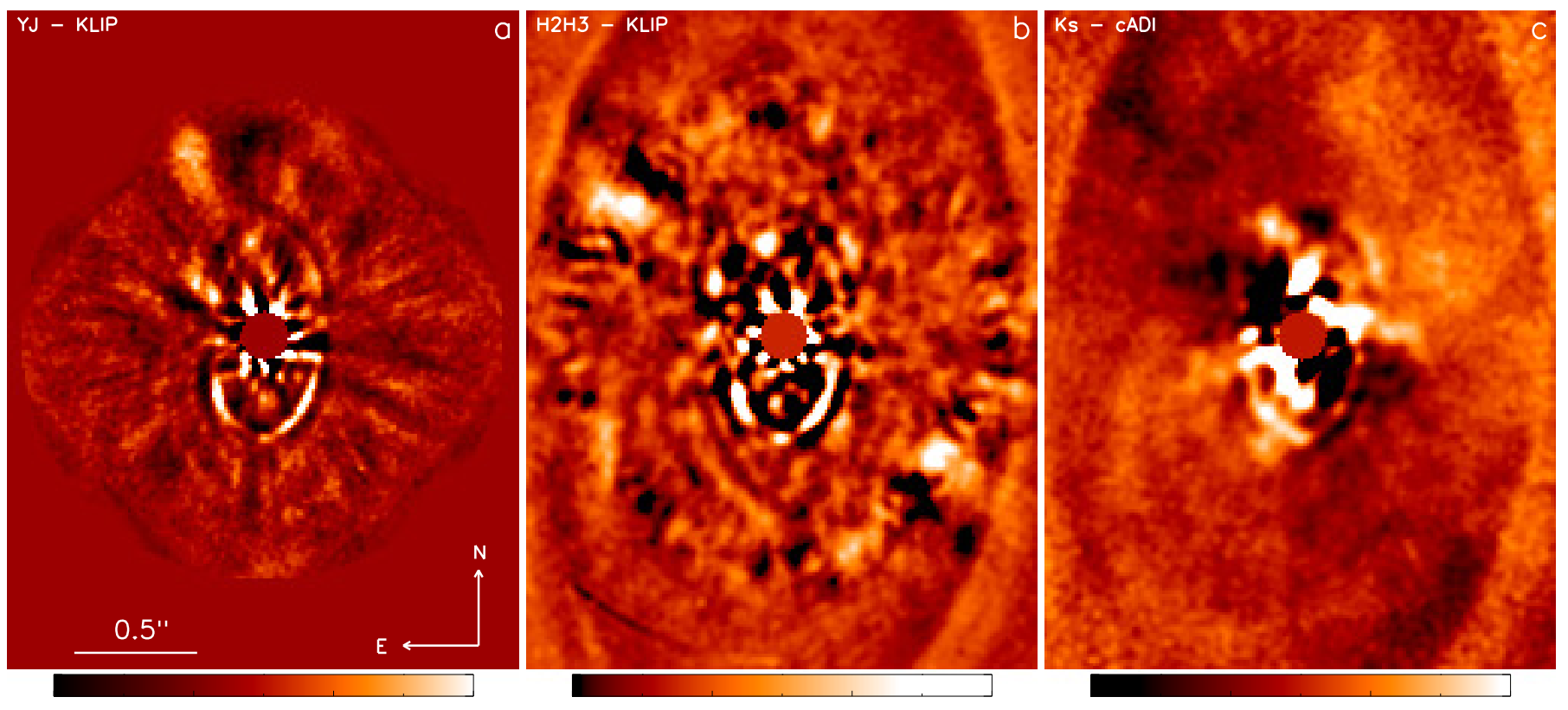

Fig. 1. Newly discovered structures in the inner region around HD141569A. Left: IFS - YJ (KLIP reduction). Middle: IRDIS - H2H3 (KLIP reduction). Right: IRDIS - Ks (cADI reduction). The cADI image is multipled by $r$ to improve the visibility of structures. East is left, north is top. See annotations of main structures in Fig. B.2.

mostly on the east side; 3) an intermediate feature (either an arc or a broken spiral arm) extending to the east from north to south in between these two rings, which clearly roots at the top of the aforementioned belt; 4) finger-like features at $1.35-1.90^{\prime \prime}$, $\mathrm{PA} \approx 148^{\circ}$; and 5 ) a very dark and steep inner cavity inwards of $1.84^{\prime \prime}(\sim 210 \mathrm{au})$.

In addition, the SPHERE images provide deep insight into the central $\sim 200$ au region. We identify several new structures that are either spiral-like or ring-like. A spiral arm S1, detected in $K \mathrm{~s}$, starts as close as $\sim 0.5^{\prime \prime}$ to the east of the star and winds to the southeast (Figs. 1, B.2i and B.1b for annotations). A more extended region is visible as a possible counterpart to the north and wrapping to the east (S2 ?, Figs. 1, B.2i and B.1b for annotations). Closer in, a series of at least three structures resembling ringlets (labelled R1 to R3, Figs. 1 and B.2 for annotations) and featuring strong asymmetries and clumpiness are discovered from both IRDIS (H2H3, Ks) and IFS (YJ) data. The $J$-band data, owing to poor observing conditions, do not achieve large enough contrasts to detect these new features. The main pattern R3 is also the brightest part of the whole disk, especially if we take into account that closer in the ADI attenuation is larger (Milli et al. 2012). The R3 pattern is strongly asymmetrical, mostly visible in the southern part while the northern part is just barely detected ( $\sim 5$ times fainter than the southern part). Surprisingly, this is orthogonal, hence, inconsistent, with the global east-west inclination of the disk, in which forwards scattering can create a brightness asymmetry with respect to the semi-major axis. We cannot posit a pericentre glow effect (Wyatt 2005) because R3 appears centred onto the star, following the north-south direction. Therefore, we suspect a true depletion of dust in this ring towards the north. The mechanisms that can cause such large azimuthal variations of the dust density remain to be studied. A clump, nearly point-like is visible in the southern ansae of R3 (PA $\left.\sim 178^{\circ}, r \sim 0.41^{\prime \prime}\right)$ surrounded by a drop of intensity on each side (Figs. 1 and B.2 for annotation), where the eastern side is darker. This feature could be a consequence of ADI artifact because a similar structure appears in forwards modelling of featureless synthetic dusty disks (see Sect. 4 and Fig. C.1). The clump and drop of intensity, however, are still detected in a spectral TLOCI reduction of the IFS data, which is not affected by the ADI bias. Moreover, the clump is slightly shifted with respect to the ansae of R3. Whether it could be associated with a real object requires more data that do not suffer from ADI biases (polarimetry for instance). Two other much fainter structures which that look like broken rings, $\mathrm{R} 1$ and $\mathrm{R} 2$, are visible at larger separations than R3 (Figs. 1 and B.2 for annotations). All these three ringlets are recurrent patterns in the various datasets YJ, H2H3, and Ks, collected at two epochs (Fig. B.2). Their elliptical shape departs from the nearly circular starlight residuals, which are particularly strong at the correction radius $\left(\sim 0.8^{\prime \prime}\right.$ in $H$-band). Given that the disk is visible in a large range of radius and azimuthal angle, calculating a $\mathrm{S} / \mathrm{N}$ map to test the reliability of R1-R3 would be impractical. Instead we plot the radial profiles of the KLIP-H2H3 deprojected image, azimuthaly averaged in four quadrants (Fig. B.3). The ringlets R3 and R2 are clearly identified as bumps localized at a constant radius. The case of R1 is more ambiguous as it appears in only two quadrants (SE and NW) and at different radii. Therefore it is not ascertained whether R1 is a ringlet or a spiral. Other fainter structures (also elliptical) may possibly exist but are not differentiable from speckles. It is yet unclear whether these three rings correspond to a nearly concentric system, which to some extent are similar to those around HL Tau (ALMA-Partnership 2015) and TW Hydrae (Rapson et al. 2015), or wherther they are hints of spiral arms.

The extended and nearly continuous disk component detected by HST's STIS instrument (Konishi et al. 2016) is not visible in the SPHERE images partly because the ADI process filters out such broad features. Instead, we are sensitive to higher frequency variations on top of this inner disk which we resolve as ringlets or spirals. The $L^{\prime}$ detection from Currie et al. (2016) partially matches with the ansa of R3 and the structures they named $\mathrm{H} 1$ and $\mathrm{H} 2$ may correspond to R2. However, they did not observe any north-south asymmetry visible at shorter 
wavelengths with SPHERE. Moreover, the point-like source reported by Currie et al. (2016; PA $\sim 180^{\circ}, R \sim 0.28^{\prime \prime}$ ) is not detected in our images (see detection limits in Sect. 5).

\subsection{Localization of the structures}

Structures were registered in a similar way as in Boccaletti et al. (2013). First, we extracted the radial profiles (azimuthally sampled by steps of $1^{\circ}$ ) of structures that are detected in several wavelengths. A one-dimensional (1D) Gaussian model is fitted on these profiles to provide the location of the maxima of the structures. These measurements for $\mathrm{R} 1, \mathrm{R} 2$, and $\mathrm{R} 3$ are reported in Fig. B.4. Assuming the ringlets are each part of an individual inclined ring, we used a non-linear least squares algorithm to fit these maxima with an elliptical contour considering a Gaussian weighting. The free parameters of the elliptical contour are the semi-major and semi-minor axes, the position angle (PA) and offsets with respect to the position of the star. We performed the fitting for each band (IRDIS $K \mathrm{~s}$ and $\mathrm{H} 2 \mathrm{H} 3$, IFS YJ) and each algorithm (cADI, TLOCI, and KLIP). Table A.2 provides averaged values and dispersions for different algorithms and wavelengths.

We found that all ringlets $\mathrm{R} 1, \mathrm{R} 2$, and $\mathrm{R} 3$ have an inclination in the range $56-58^{\circ}$ compatible with the inclination found for the inner ring $\left(56.9 \pm 1.0^{\circ}\right)$ within error bars. The PAs of R2 and R3 are slightly different than the global orientation of the disk by $\sim 1-2^{\circ}$. Finally, we measured offsets of $15.4 \pm 3.4$ mas $(1.79 \pm$ $0.40 \mathrm{au}), 15.4 \pm 4.8$ mas $(1.79 \pm 0.56 \mathrm{au})$, and $34.9 \pm 5.1 \mathrm{mas}$ $(4.05 \pm 0.59 \mathrm{au})$ towards west, respectively for R3, R2, and R1, plus an offset of $82.2 \pm 17.0$ mas $(9.54 \pm 1.97 \mathrm{au})$ towards north for R1. In addition, the inner ring at 210 au has an opposite offset direction. We measured $29.8 \pm 7.8$ mas $(3.46 \pm 0.90 \mathrm{au})$ towards east and $32.8 \pm 6.9$ mas $(3.80 \pm 0.80 \mathrm{au})$ towards north in agreement with Mazoyer et al. (2016). Here we did not considered ringlets ellipticity even though could account for the differential offset. In particular, R1 has an important offset (Table A.2). As explained above, however, the exact nature of R1 (ringlet or spiral) is left undetermined.

Considering the linear wave density theory (Rafikov 2002) and the tools we previously developed (Boccaletti et al. 2013), we attempted to fit the spiral feature S1 on the deprojected disk image. We did not find a set of parameters which produces a match between the model and S1 using a simpler Archimedean spiral model either. A more sophisticated model might be required to account for the shape of $\mathrm{S} 1$ if produced by a planet (Dong et al. 2015b). Alternatively, the system could have experienced gravitational instabilities (Dong et al. 2015a), or the spiral arm could be in a different plane than the other parts of the disk, as suggested in Biller et al. (2015).

\section{Photometry}

We performed disk modelling and photometry of the ringlet $\mathrm{R} 3$ to derive qualitative results (a thorough modelling is postponed to future work). Following earlier work (Boccaletti et al. 2012; Mazoyer et al. 2014), we built a set of geometrical disks model using GRaTer (Augereau et al. 1999b) with no particular assumption about the grain optical properties. To restrain the parameter space we fixed the PA of the ringlet to $356.5^{\circ}$ (compatible with the fit previously described). The inclination $i$ and the position of the ring $\left(r_{0}\right)$ span a narrow range of values $(i=57$, $\left.58,59^{\circ} ; r_{0}=47,48,49 \mathrm{au}\right)$ since R3 is already well defined in the images. The model assumes that the surface density radially decreases inwards and outwards of $r_{0}$ as power laws with slopes $\alpha_{\text {in }}$ and $\alpha_{\text {out }}$, respectively. We used a large range of values for these parameters: 2, 5, 10 and 20 for $\alpha_{\text {in }}$ and $-2,-5,-10$ and -20 for $\alpha_{\text {out }}$. We set the aspect ratio to $h / r=0.01$ (following Thi et al. 2014), where $r$ is the separation to the star and $h$ is the height of the disk. Moreover, we assumed a front-back (eastwest) symmetrical ring with isotropic scattering $(g=0)$ as we are interested in the southern part of R3 alone. We ran the least squares minimization between the grid of models and the IRDIS H2H3 KLIP image. The best model (minimum $\chi^{2}$ ) yields $i=57^{\circ}$ and $r_{0}=48$ au $\left(0.41^{\prime \prime}\right)$, which are both in close agreement with the ellipse fitting, and surface density slopes of $\alpha_{\text {in }}=20$ and $\alpha_{\text {out }}=-20$. Considering a more conservative threshold accounting for the degree of freedom in the fitting procedure, we end up with a list of possible models all having surface density slopes modulus of 10 or 20. Similar results are derived from model fitting in the $K$ s-band. We can conclude that the ringlet $\mathrm{R} 3$ is rather narrow (radially unresolved) and has very sharp edges both inwards and outwards (Fig. C.1), which implies that it is probably bounded by perturber(s) or shaped by the coupling of gas and dust as described for example in Lyra \& Kuchner (2013).

Using the best model to estimate the ADI bias, we measured the integrated intensity in the southern part of R3 to be $0.45 \mathrm{mJy}$ and $0.35 \mathrm{mJy}$ in the $H$ and $K \mathrm{~s}$-bands, respectively. These numbers stand for rough estimations, but are consistent with the variation of the flux of the star from $H$ to $K \mathrm{~s}$, as we should expect for scattering.

In addition, a simple radiative transfer toy model was developed to test the geometry probed by SPHERE observations against the VISIR images shown in Thi et al. (2014). This toy model is based on a set of concentric rings for which the radii are set according to the angularly resolved existing images in scattered light: 380-420 au, 190-210 au, and the newly found 45-48 au ringlets (R3). Using only this set of three concentric rings, the resulting profile at $8.6 \mu \mathrm{m}$ is inconsistent with VISIR $8.6 \mu \mathrm{m}$ image. The thermal flux in the inner 0.2" (equivalent to the VISIR resolution) is not large enough compared to what is observed. In the mid-IR an additional component is thus required closer to the star. Given VISIR resolution, any additional ring-shaped component with a mean radius smaller than $\sim 20$ au would be compatible with VISIR data.

\section{Detection limits}

While the many structures of the transition disk HD 141569A suggest the presence of planets, we do not detect any reliable point sources, apart from speckles, which are present inside $1^{\prime \prime}$ (for instance at $r \sim 0.38^{\prime \prime}, \mathrm{PA} \sim 11^{\circ}$ and $r \sim 0.35^{\prime \prime}, \mathrm{PA} \sim 51^{\circ}$ ). Hence, we measured the contrast, at $5 \sigma$, in $J, \mathrm{H} 2 \mathrm{H} 3, K$ s and $\mathrm{YJ}$ (Fig. D.1) bands for data processed with TLOCI (optimized for point sources). The ADI throughput is accounted for by a customized pipeline, SpeCal. From these detection limits, we converted in Jupiter mass (Fig. 2), assuming the latest evolutionary model (BHAC-2015 + COND-2003, Baraffe et al. 2003). The IFS YJ and IRDIS H2H3 contrasts are far superior to the other bands as a result of data quality. For an arbitrary separation of $0.5^{\prime \prime}$ (roughly where the detection limit in mass starts to flatten), the $\mathrm{H} 2$ (respectively $K \mathrm{~s}$ ) image would have allowed the detection of 1-2 $M_{\mathrm{J}}$ (respectively, 2-3 $M_{\mathrm{J}}$ ). The limit in $J$-band is worse inside $0.6^{\prime \prime}$ but then similar to the limit in $K$ s outwards. We found no planets more massive than $1-3 M_{\mathrm{J}}$, between $0.3^{\prime \prime}$ (ringlet R3) and $1.84^{\prime \prime}$ (the edge of the inner belt). Inside the radius of R3 the detection performance degrades rapidly to about $10 M_{\mathrm{J}}$ near the inner working angle (IWA) of the coronagraph (93 mas). At the PA and separation of the point-like source reported by Currie et al. (2016; estimated to 5-6 $M_{\mathrm{J}}$ ), and not observed in 


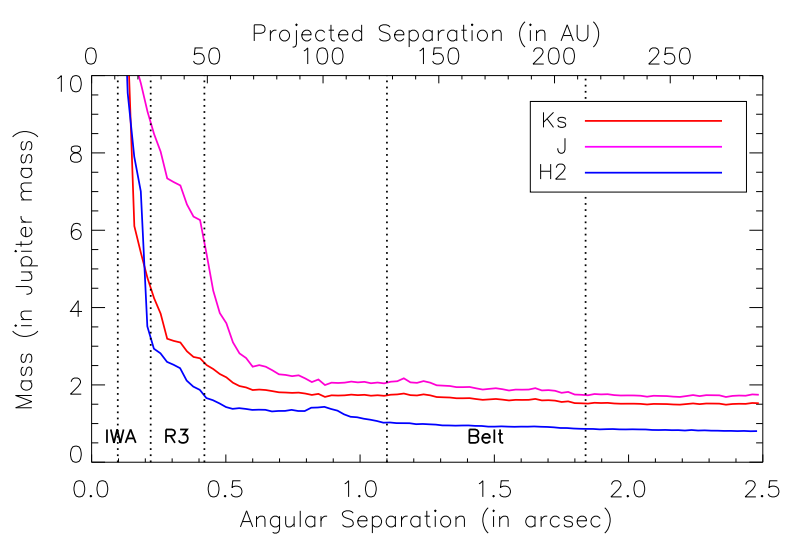

Fig. 2. Detection limit in Jupiter mass for $J, \mathrm{H} 2$ and $K$ s-band, assuming the BHAC-2015+COND-2003 model. IWA is the inner working angle of the coronagraph. R3 and Belt represent the separation where the structures are located.

our image, our data yield a detection limit of $3.5 M_{\mathrm{J}}$ in $K \mathrm{~s}$ and $2.5 M_{\mathrm{J}}$ in $\mathrm{H} 2$, hence, this limit is not compatible with the mass derived from $L^{\prime}$.

\section{Conclusion}

Exploring the inner $1^{\prime \prime}$ region with SPHERE of the transition disk HD 141569A has revealed a series of concentric ringlets at physical separation of $47 \mathrm{au}, 64$ au and 93 au, partially associated with the emission formerly detected by the VISIR instrument (Thi et al. 2014). An additional dust component closer than $0.2^{\prime \prime}$ may be required to account for this emission. However, these new structures match perfectly the extended disk component found by STIS (Konishi et al. 2016) and the north-south extension by Currie et al. (2016). We have shown that the inclination and the PA of each of these ringlets are in perfect agreement with those of the outer belts. The brightest ringlet is so asymmetrical that it appears as a half-ring. This brightness asymmetry is not consistent with forwards scattering and may then be the result of a true azimuthal variation of the dust density; the reason for this has yet to be understood. We also noted the presence of a clump in the south of the brighter ringlet, which could be an artifact from the reduction process or a local variation of the dust density. In addition, it is difficult to trace each of these broken rings exactly in all directions hence, there is a possible confusion with spiral patterns. A large spiral pattern is observed in $K$ s, developing southwest with possibly a northern counterpart. These observed structures could be the consequence of perturbations by planets, which confine dust grains and create gaps. However, we found no planets more massive than 1-3 $M_{\mathrm{J}}$ between the edge of the cavity and the ringlet R3. These kinds of structures could also be created by the coupling of gas and dust triggering instabilities in the form of narrow eccentric rings when the gas-dust ratio is close to the unity (Lyra \& Kuchner 2013).

Acknowledgements. We acknowledge financial support from the Programme National de Planétologie (PNP) and the Programme National de Physique Stellaire (PNPS) of CNRS-INSU. This work has also been supported by a grant from the French Labex OSUG@2020 (Investissements d'avenir - ANR10 LABX56). The project is supported by CNRS, by the Agence Nationale de la Recherche (ANR-14-CE33-0018). This work is partly based on data products produced at the SPHERE Data Centre hosted at OSUG/IPAG, Grenoble. We thank P. Delorme and E. Lagadec (SPHERE Data Centre) for their efficient help during the data reduction process. SPHERE is an instrument designed and built by a consortium consisting of IPAG (Grenoble, France), MPIA (Heidelberg, Germany), LAM (Marseille, France), LESIA (Paris, France), Laboratoire
Lagrange (Nice, France), INAF-Osservatorio di Padova (Italy), Observatoire de Genève (Switzerland), ETH Zurich (Switzerland), NOVA (Netherlands), ONERA (France) and ASTRON (Netherlands) in collaboration with ESO. SPHERE was funded by ESO, with additional contributions from CNRS (France), MPIA (Germany), INAF (Italy), FINES (Switzerland) and NOVA (Netherlands). SPHERE also received funding from the European Commission Sixth and Seventh Framework Programmes as part of the Optical Infrared Coordination Network for Astronomy (OPTICON) under grant number RII3-Ct-2004-001566 for FP6 (2004-2008), grant number 226604 for FP7 (2009-2012) and grant number 312430 for FP7 (2013-2016). V.C. acknowledges support from the European Research Council under the European Union's Seventh Framework Programme (ERC grant agreement No. 337569) and from the French Community of Belgium through an ARC grant for Concerted Research Action. J. O. acknowledges support from the Millennium Nucleus RC130007 (Chilean Ministry of Economy).

\section{References}

ALMA-Partnership 2015, ApJ, 808, L3

Ardila, D. R., Lubow, S. H., Golimowski, D. A., et al. 2005, ApJ, 627, 986 Augereau, J. C., \& Papaloizou, J. C. B. 2004, A\&A, 414, 1153

Augereau, J. C., Lagrange, A. M., Mouillet, D., \& Menard, F. 1999a, A\&A, 350, L51

Augereau, J. C., Lagrange, A. M., Mouillet, D., Papaloizou, J. C. B., \& Grorod, P. A. 1999 b, A\&A, 348, 557

Baraffe, I., Chabrier, G., Barman, T. S., Allard, F., \& Hauschildt, P. H. 2003, A\&A, 402, 701

Beuzit, J.-L., Feldt, M., Dohlen, K., et al. 2008, in SPIE Conf. Ser., 7014, 18

Biller, B. A., Liu, M. C., Rice, K., et al. 2015, MNRAS, 450, 4446

Boccaletti, A., Abe, L., Baudrand, J., et al. 2008, in SPIE Conf. Ser., 7015, 1

Boccaletti, A., Augereau, J. C., Lagrange, A. M., et al. 2012, A\&A, 544, A85

Boccaletti, A., Pantin, E., Lagrange, A. M., et al. 2013, A\&A, 560, A20

Boccaletti, A., Thalmann, C., Lagrange, A.-M., et al. 2015, Nature, 526, 230

Brittain, S. D., Rettig, T. W., Simon, T., et al. 2003, ApJ, 588, 535

Clampin, M., Krist, J. E., Ardila, D. R., et al. 2003, ApJ, 126, 385

Claudi, R. U., Turatto, M., Gratton, R. G., et al. 2008, in SPIE Conf. Ser., 7014

Currie, T., Grady, C. A., Cloutier, R., et al. 2016, ApJ, 819, L26

Dent, W. R. F., Greaves, J. S., \& Coulson, I. M. 2005, MNRAS, 359, 663

Dohlen, K., Langlois, M., Saisse, M., et al. 2008, in SPIE Conf. Ser., 70143

Dong, R., Hall, C., Rice, K., \& Chiang, E. 2015a, ApJ, 812, L32

Dong, R., Zhu, Z., Rafikov, R. R., \& Stone, J. M. 2015b, ApJ, 809, L5

Dutrey, A., Lecavelier Des Etangs, A., \& Augereau, J. C. 2004, Comets II, 81

Fusco, T., Sauvage, J.-F., Petit, C., et al. 2014, in SPIE Conf. Ser., 91481 U

Garufi, A., Quanz, S. P., Schmid, H. M., et al. 2014, A\&A, 568, A40

Goto, M., Usuda, T., Dullemond, C. P., et al. 2006, ApJ, 652, 758

Konishi, M., Grady, C. A., Schneider, G., et al. 2016, ApJ, 818, L23

Lafrenière, D., Marois, C., Doyon, R., Nadeau, D., \& Artigau, É. 2007, ApJ, 660, 770

Langlois, M., Vigan, A., Dohlen, K., et al. 2014, in SPIE Conf. Ser., 9147, 9

Lyra, W., \& Kuchner, M. 2013, Nature, 499, 184

Maire, A.-L., Bonnefoy, M., Ginski, C., et al. 2016, A\&A, 587, A56

Marois, C., Lafrenière, D., Doyon, R., Macintosh, B., \& Nadeau, D. 2006a, ApJ, 641, 556

Marois, C., Lafrenière, D., Macintosh, B., \& Doyon, R. 2006b, ApJ, 647, 612

Marois, C., Correia, C., Galicher, R., et al. 2014, in SPIE, 9148, 91480U

Mazoyer, J., Boccaletti, A., Augereau, J. C., et al. 2014, A\&A, 569, A29

Mazoyer, J., Boccaletti, A., Choquet, É., et al. 2016, ApJ, 818, 150

Merìn, B., Montesinos, B., Eiroa, C., et al. 2004, A\&A, 419, 301

Milli, J., Mouillet, D., Lagrange, A.-M., et al. 2012, A\&A, 545, A111

Mouillet, D., Lagrange, A. M., Augereau, J. C., \& Menard, F. 2001, A\&A, 372, L61

Pavlov, A., Möller-Nilsson, O., Feldt, M., et al. 2008, in SPIE Conf. Ser., 7019, 39

Péricaud, J., di Folco, E., Dutrey, A., et al. 2015, in Young Stars \& Planets Near the Sun, eds. J. H. Kastner, B. Stelzer, \& S. A. Metchev, IAU Symp., 314, 201

Rafikov, R. R. 2002, ApJ, 569, 997

Rapson, V. A., Kastner, J. H., Millar-Blanchaer, M. A., \& Dong, R. 2015, ApJ, 815, L26

Reche, R., Beust, H., \& Augereau, J. C. 2009, A\&A, 493, 661

Soummer, R., Pueyo, L., \& Larkin, J. 2012, ApJ, 755, L28

Thi, W. F., Pinte, C., Pantin, E., et al. 2014, A\&A, 561, A50

van Leeuwen, F. 2007, A\&A, 474, 653

Vigan, A., Moutou, C., Langlois, M., et al. 2010, MNRAS, 407, 71

Weinberger, A. J., Becklin, E. E., Schneider, G., et al. 1999, ApJ, 525, L53

Wyatt, M. C. 2005, A\&A, 440, 937 


\section{Appendix A: Observing log and fit tables}

Table A.1. Observing log.

\begin{tabular}{|c|c|c|c|c|c|c|c|c|c|}
\hline Programme & Instrument & Filter & $\begin{array}{l}\lambda_{c} \\
(\mu \mathrm{m})\end{array}$ & $\begin{array}{l}\text { Date } \\
\text { UT }\end{array}$ & $\begin{array}{l}\text { FoV Rotation } \\
\left({ }^{\circ}\right)\end{array}$ & $\begin{array}{l}T_{\exp } \\
(\mathrm{s})\end{array}$ & $\begin{array}{l}\text { DIT } \\
\text { (s) }\end{array}$ & $N_{\text {exp }}$ & $\begin{array}{l}\text { TN } \\
\left(^{\circ}\right)\end{array}$ \\
\hline 095.C-0298 & IRDIS & $\mathrm{H} 2$ & 1.593 & $2015-05-16$ & 42.07 & 4096 & 64 & 64 & $-1.8 \pm 0.1$ \\
\hline 095.C-0298 & IRDIS & H3 & 1.667 & $2015-05-16$ & 42.07 & 4096 & 64 & 64 & $-1.8 \pm 0.1$ \\
\hline 095.C-0298 & IFS & YJ & $0.95-1.35$ & 2015-05-16 & 42.07 & 4096 & 64 & 64 & $-1.8 \pm 0.1$ \\
\hline 095.C-0381 & IRDIS & $J$ & 1.245 & $2015-07-22$ & 34.89 & 3200 & 8 & 400 & $-1.67 \pm 0.03$ \\
\hline 095.C-0381 & IRDIS & $K \mathrm{~s}$ & 2.182 & 2015-07-28 & 35.54 & 3200 & 16 & 200 & $-1.67 \pm 0.03$ \\
\hline
\end{tabular}

Notes. The table includes: programme name, Instrument, acquisition mode and filter, central wavelength, date, variation of parallactic angle, total exposure time, individual exposure time, number of frames, true North calibration.

Table A.2. Parameters for the ringlets assuming offset ellipses.

\begin{tabular}{lllllll}
\hline \hline Structure & $\begin{array}{l}\text { Semi-major axis } \\
(\mathrm{mas})\end{array}$ & $\begin{array}{l}\text { Semi-minor axis } \\
(\mathrm{mas})\end{array}$ & $\begin{array}{l}\text { PA } \\
\left({ }^{\circ}\right)\end{array}$ & $\begin{array}{l}\text { Inclination } \\
\left({ }^{\circ}\right)\end{array}$ & $\begin{array}{l}\text { West offset } \\
(\mathrm{mas})\end{array}$ & $\begin{array}{l}\text { North offset } \\
(\mathrm{mas})\end{array}$ \\
\hline Inner ring & $1774.8 \pm 11.2$ & $970.1 \pm 18.6$ & $356.1 \pm 0.4$ & $56.9 \pm 1.0$ & $-29.8 \pm 7.8$ & $32.8 \pm 6.9$ \\
R1 & $805.0 \pm 14.8$ & $431.2 \pm 7.9$ & $356.0 \pm 2.0$ & $57.6 \pm 1.3$ & $34.9 \pm 5.1$ & $82.2 \pm 17.0$ \\
R2 & $549.1 \pm 12.1$ & $307.0 \pm 11.0$ & $354.5 \pm 1.0$ & $56.0 \pm 2.2$ & $15.4 \pm 4.8$ & $5.8 \pm 4.8$ \\
R3 & $406.2 \pm 7.2$ & $215.8 \pm 3.8$ & $353.7 \pm 1.1$ & $57.9 \pm 1.3$ & $15.4 \pm 3.4$ & $1.2 \pm 9.4$ \\
\hline
\end{tabular}

\section{Appendix B: Multi-views and annotations of HD 141569A disk}
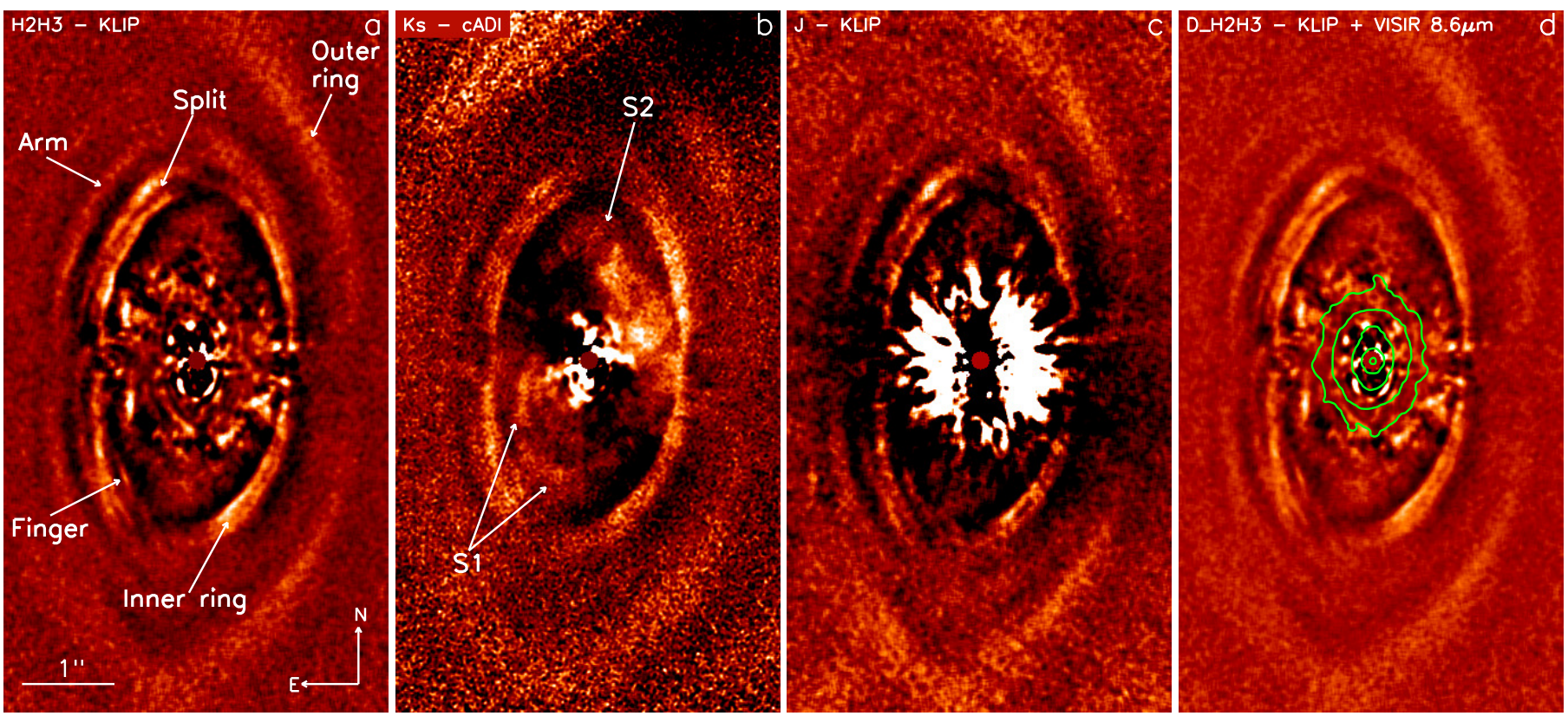

Fig. B.1. Reduced images of the disk around HD 141569A. a) Wide field of view obtained in H2H3 (KLIP reduction, annotation from Mazoyer et al. 2016). b) cADI reduction of $K$ s image with the annotation of the spirals S1 and S2. c) KLIP reduction of $J$ image. d) KLIP reduction of $\mathrm{H} 2 \mathrm{H} 3$ image with the VISIR contour at $8.6 \mu \mathrm{m}$. All images are arbitrarily multiplied by the distance to the star in pixel for cosmetics reason. The spatial scale is the same for the four images. East is left, north is up. 
C. Perrot et al.: Discovery of concentric broken rings inside $1^{\prime \prime}$ in the transition disk HD 141569A with VLT/SPHERE
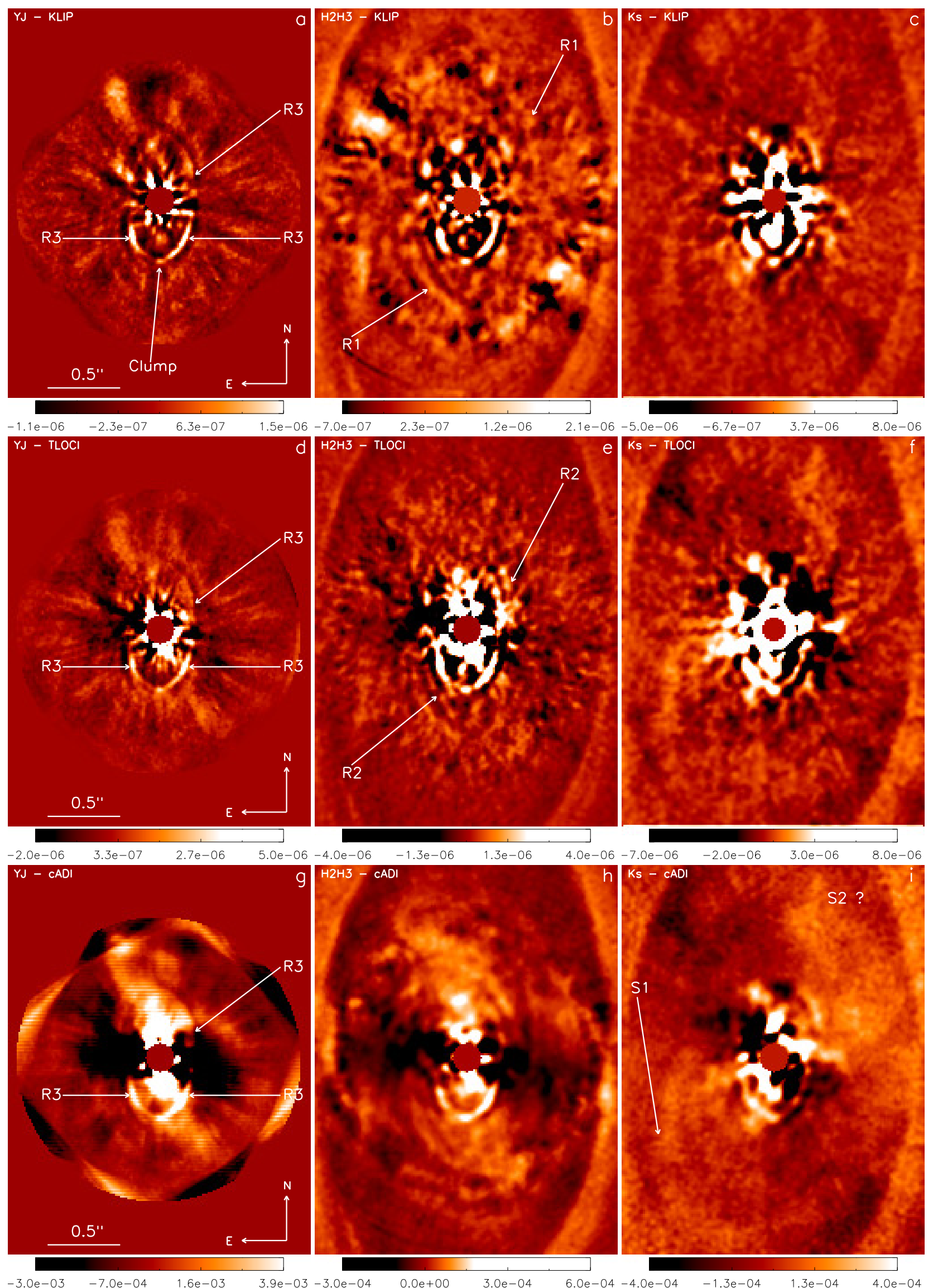

Fig. B.2. Images of the central part of the system in YJ (left column), H2H3 (middle column), and Ks (right column) bands with three differents post-processing: KLIP (top row), TLOCI (middle row), and cADI (bottom row). The cADI images are multipled by $r$ to improve the visibility of structures. The annotations indicate structures R1, R2, R3, S1-S2, and the clump for a better identification. 


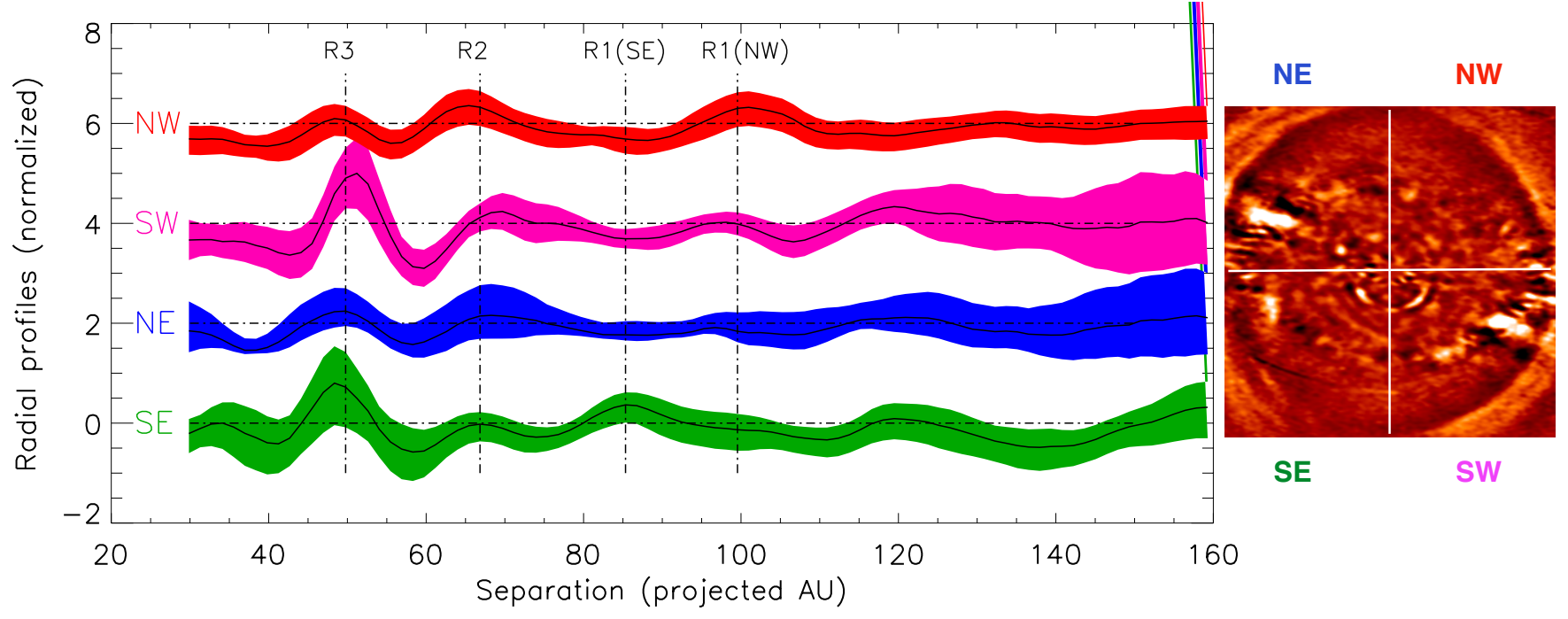

Fig. B.3. Radial profiles of the KLIP IRDIS-H2H3 deprojected image as measured in four quadrants shown in the right panel (red: north-west, magenta: south-west, blue: north-east and green: south-east). Black lines stand for averaged profile and the colour shaded areas indicate the azimuthal dispersion. All profiles are normalized and vertically shifted for the sake of clarity.

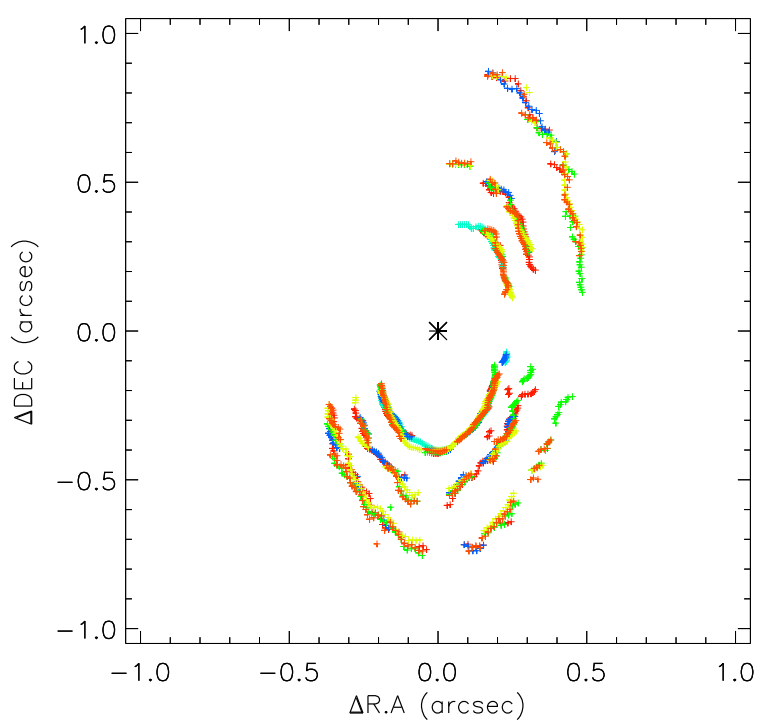

Fig. B.4. Registration of the ringlets in the IRDIS H2H3 and $K$ s-band images for several algorithms (KLIP, TLOCI and cADI). $K \mathrm{~s}-\mathrm{KLIP}$ (red), Ks - cADI (cyan), Ks - TLOCI (blue), H2H3 - KLIP (green), H2H3 - cADI (yellow), and H2H3 TLOCI (orange). 


\section{Appendix C: Models compared to data}
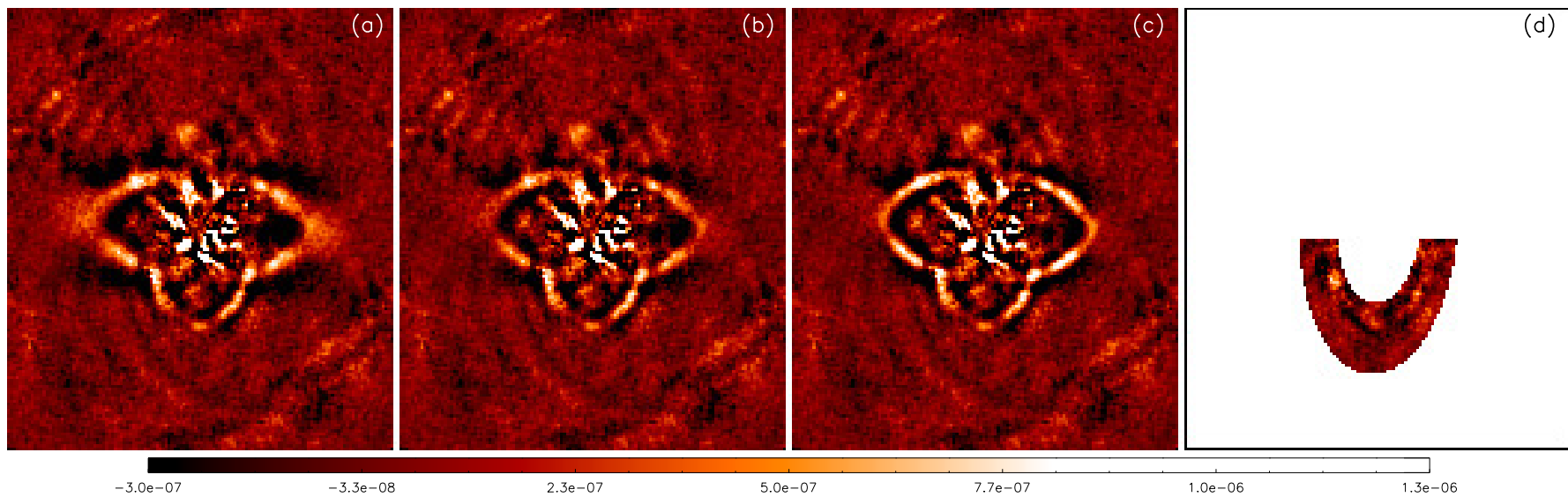

$-3.3 e-08$

$2.3 e-07$

$5.06-07$

$1.0 e-06$

$1.3 e-06$

Fig. C.1. Comparison between the $\mathrm{H} 2 \mathrm{H} 3$ image (vertical component) and disk models (horizontal component) with different values of $\alpha_{\text {in }}$ and $\alpha_{\text {out }}$ a) $\alpha_{\text {in }}=5$ and $\alpha_{\text {out }}=-5 ;$ b) $\alpha_{\text {in }}=10$ and $\alpha_{\text {out }}=-10 ;$ c) $\alpha_{\text {in }}=20$ and $\alpha_{\text {out }}=-20$. The inclination is set to $58^{\circ}$ and the semi-major axis is set to $0.41^{\prime \prime}$. Panel d) shows the residuals after subtraction of the best model $\left(\alpha_{\mathrm{in}}=20\right.$ and $\left.\alpha_{\text {out }}=-20\right)$.

\section{Appendix D: Contrast limits for point source}

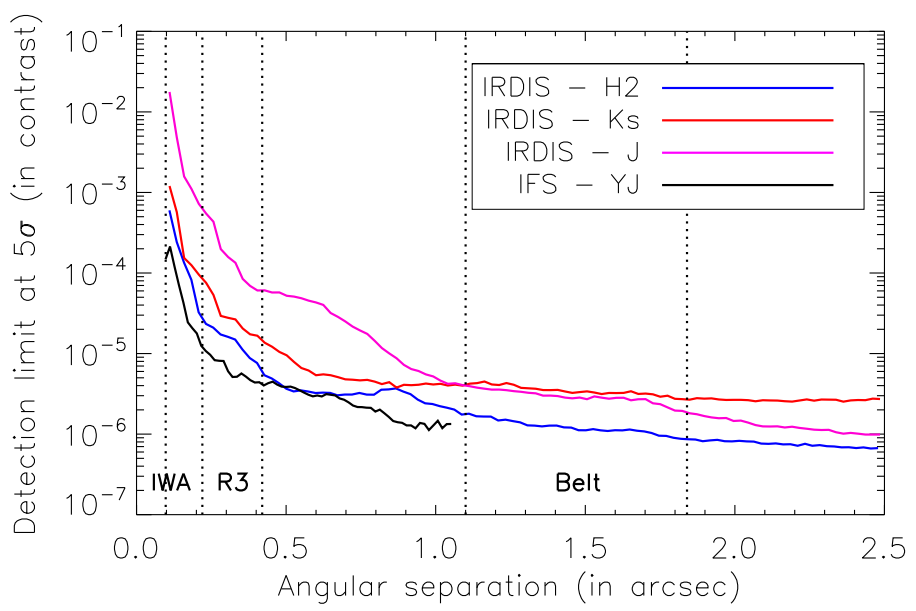

Fig. D.1. Detection limit in contrast for YJ, $J, \mathrm{H} 2$, and $K$ s-bands with the TLOCI images. The contrast is obtained by an azimuthal standard deviation for each angular separation, corrected by the throughput (SpeCal, R. Galicher, priv. comm.). 